\title{
Ethical issues for e-cigarette control policies in Australia
}

\author{
SOUMYADEEP BHAUMIK
}

\begin{abstract}
Although tobacco smoking in Australia is at a historical low, electronic cigarette (e-cigarette) use, especially among the youth is increasing. Policies around e-cigarette control in Australia are currently evolving, even during the pandemic, thus demonstrating its priority status. The current article discusses ethical issues for e-cigarette control policies in Australia using a public health ethics framework. The article is structured using the domains of the WHO-MPOWER framework of tobacco control to enable a comprehensive coverage of all elements of ecigarette control policies in Australia. It highlights several ethical issues, from different stakeholder perspectives, and indicates moral and ethical tensions in different public health actions that might be considered in framing policies around e-cigarette control.
\end{abstract}

Keywords: Electronic nicotine delivery systems, e-cigarettes, Australia,, smoke-free policy, public health ethics

\section{Introduction}

Australia has seen a sustained decrease in smoking for many decades by implementing a comprehensive multi-level tobacco control strategy $(1,2)$. The proportion of Australians who smoke tobacco daily has decreased from $24 \%$ in 1991 to $12.2 \%$ in 2016 , and $11.0 \%$ in 2019(3). However, it is seen that proportion of Australians who have ever used electronic cigarettes (e-cigarettes) has increased from $8.8 \%$ in 2016 to $11.3 \%$ in 2019 (3). The policy and regulatory space around ecigarette is currently evolving in Australia, even amidst the Covid-19 pandemic. Policy critique and analysis of evidence

Author: Soumyadeep Bhaumik (sbhaumik@georgeinstitute.org.in), The George Institute for Global Health, Faculty of Medicine, University of New South Wales, Sydney, AUSTRALIA and The George Institute for Global Health, Meta-research and Evidence Synthesis Unit, Elegance Tower, New Delhi 110 025, INDIA.

To cite: Bhaumik S. Ethical issues for e-cigarette control policies in Australia. Indian J Med Ethics. Published online on July 24, 2021. DOI: 10.20529/IJME. 2021.055.

Manuscript Editor:Vijayaprasad Gopichandran

Peer Reviewers: C Priyadarshini and an anonymous reviewer

(c) Indian Journal of Medical Ethics 2021 on e-cigarettes is substantial (4-8), but analysis of the ethical issues in Australia is scant. Previous analysis of ethical issues control e-cigarettes in Australia has been either based on principlism (the ethical principles of autonomy, beneficence, non-maleficence, and justice) $(9,10)$ or through the prism of harm reduction (10-12). Principlism is not well suited to analysing ethical issues around public health policies. Public health decision making is inherently more complex, involves larger numbers of stakeholders with different value systems, and is contextual in nature. Harm reduction, though a key tenet to guide e-cigarette policies, has its roots in libertarianism, a value system which might fundamentally not align with the moral beliefs of many stakeholders. As such, using a single philosophical lens for analysing public policy is not desirable.

This article analyses ethical issues around all aspects of ecigarette control in Australia using a three-step public health ethics framework (13). The three-step framework does not presume superiority of any set of moral norms over another and helps clarify ethical issues contextually and comprehensively. The approach in brief consists of analysing ethics and contexts around the issue at hand, analysing ethical dimensions of alternative courses of action and a final stage of justification for a particular public health decision. The need for a comprehensive policy framework to tackle ecigarette use has been identified (14), and as such, an ethical analysis of all aspects of control is necessary. To ensure comprehensive coverage of e-cigarette control policies, the ethical analysis is structured within the World Health Organization's WHO-MPOWER framework of tobacco control (15). The WHO-MPOWER (Monitor tobacco use and prevention policies, Protect people from tobacco smoke, Offer help to quit tobacco use, Warn about the dangers of tobacco, Enforce bans on tobacco advertising, promotion and sponsorship, Raise taxes on tobacco) is a comprehensive framework to monitor implementation of control policies (16). To the best of my knowledge, such a comprehensive approach for understanding ethical issues around e-cigarette control policies has not been undertaken globally.

\section{Monitoring of e-cigarette use and prevention}

The Australian Institute of Health and Welfare, which periodically conducts surveys for monitoring drug and tobacco use, also provides data on e-cigarette use (3). However, estimates of smoking and e-cigarette use in the 
Aboriginal and Torres Strait Islanders (indigenous) people, and migrant communities is not available from these surveys. Tensions around equity, justice and respect for indigenous people and their cultural practices dominate a large part of the socio-political discourse in Australia. The Australian Bureau of Statistics (ABS), which conducts surveys under the Census and Statistics Act, 1905, provided the first government estimate in 2019 and found that the ever-use of e-cigarette(had used e-cigarettes ever in their lifetime) was $8 \%$ in indigenous adult people (17). A 2020 review commissioned by the Australian Government noted the unique risk for indigenous people due to the entrenching of colonial processes and commercialisation within their communities, in the context of the diverse and evolving range of e-cigarette products being available (18).

Having a contextually fit equity-focussed monitoring framework which looks at monitoring of use and prevention of e-cigarettes in indigenous and migrant communities, with further disaggregation by gender and region (rural/urban) might be considered by the Australian government. As such Australia, has an opportunity to go above and beyond international commitments under the WHO FCTC (16) by considering equity within its tobacco control related surveys. It is expected that this will incur costs as larger sample size surveys will be required to get reliable estimates. A distributive justice framework would justify the additional investments required for this purpose.

\section{Protecting people from e-cigarette smoke}

The deleterious effect of second-hand smoke emanating from e-cigarettes, and the consequent need to protect people is intensely debated. Proponents of e-cigarettes (manufacturers, retailers, trade associations, hospitality industry and a section of researchers and liberal politicians) cite data on e-cigarette smoke having less nicotine than cigarettes (or none for non-nicotine varieties), and on the uncertainty around harms from vapour emanating from second-hand e-cigarette smoke (19). A key ethical principle invoked is the liberal tenet of Mill's harm principle (20), which broadly states that people's autonomy should be preserved unless it causes harm to others. Those opposing e-cigarettes (tobacco and cancer control activists and peak medical bodies $^{1}$ ) cite emerging data on the harms from second-hand smoke. They call for invoking the precautionary principle and banning e-cigarette use in public spaces, like the measures against cigarette smoking (21).

The "gateway effect", ie e-cigarette use leading to initiation of smoking in non-smokers (discussed subsequently) and the renormalisation of the culture of smoking have also been cited as harms which should be considered $(10,12)$. As such, Mill's harm principle has been challenged on moral grounds citing the potential harms for prospective smokers. Proponents of e-cigarettes argue that interests of a hypothetical future smoker cannot trump the autonomy of current smokers (9). It is illegal to use e-cigarettes (irrespective of whether they contain nicotine) in places where smoking is banned, ie in almost all states and territories of Australia currently.

\section{Offering help for smoking cessation through e- cigarettes}

Current Australian guidelines do not recommend e-cigarettes as either first-line or second line therapy, noting the lack of evidence on effectiveness and safety for smoking cessation (22). However, the October 2020 Cochrane review (23), found that quit rates were higher (moderate certainty evidence) and there was no difference in adverse events (low-certainty evidence) in people who used e-cigarettes containing nicotine in comparison to those who used other NRT, or those who use non-nicotine e-cigarettes.

Away from the clinical debates around effectiveness, it has also been argued that use of nicotine-containing e-cigarettes as an option makes smoking cessation messages confusing for the public $(9,24)$. The public might possibly interpret it as evidence for a safe level of smoking. The additional harm due to dual use of cigarettes and e-cigarettes, becoming a deterrent to quitting tobacco altogether is an important cause of concern. However, such an argument is broadly true for any harm reduction approach including NRT. Such an argument reflects a pre-existing biased moral reasoning against e-cigarette (24) as a harm-reduction approach.

Selling e-cigarettes containing nicotine is already banned throughout Australia, under the National Poisons Standard. In September 2020, the Therapeutic Goods Administration (TGA) made an interim decision to make it a "prescription only" product for those needing it for smoking cessation (25). This follows a decision in June 2020, wherein importing ecigarettes containing nicotine (or their refills) was banned in Australia from January 1, 2021 (26). This implies that, individuals can no longer order online and/or bring nicotine containing e-cigarettes by international surface or airmail services. Medical practitioners will also have to register themselves separately to prescribe nicotine containing ecigarettes. The process is thought to be a significant barrier especially in the context wherein e-cigarettes are not recommended as smoking cessation therapy in guidelines (22). Non-nicotine e-cigarettes, however, continue to be available for local purchase, with little restriction in most Australian states and territories, although these contain several other potentially harmful substances.

In Australia, smoking is currently more prevalent in socioeconomically disadvantaged groups (3). Many proponents of e-cigarettes argue that the barriers for e-cigarette use for smoking cessation is an issue of inequity. They argue it is "unfair to addicted smokers who are denied access to a safer nicotine product and forced to continue to smoke cigarettes" (9). Evidence from some observational studies show that some cigarette smokers who are not motivated to quit otherwise found e-cigarettes helpful for cessation (19). Some politicians have claimed that those against e-cigarettes 
are "in the pocket of tobacco companies" (27) since the tobacco industry is losing business due to rising e-cigarette use. While these are unsubstantiated claims, the influence and interest of the tobacco industry in promoting e-cigarette use globally has been well documented and is discussed subsequently.

Considering the evidence from the recent Cochrane review (23), there seems to be a possible therapeutic role for ecigarettes in reducing smoking, should the guidelines change. Retailers and convenience store owners in general object to the regulation of e-cigarettes being harsher than that of cigarettes (principle of justice). They also cite the need to make-up for decreasing cigarette sales and for losses due to the Covid-19 pandemic, as reason for dependency on ecigarettes (28). Policy stances around the issue, both between and within stakeholder groups, are likely to remain fluid as the economic impact of the pandemic unfolds.

\section{Warning about the dangers of e-cigarettes}

E-cigarettes which do not contain nicotine are sold with minimal to no retail restrictions in most Australian states. The ambiguity around safety and long-term implications of ecigarette use is not communicated to e-cigarette users as there are no standardised warnings on the issue. Several peak medical bodies in their submission to a government enquiry on e-cigarettes in 2017 have called for legal requirements in packing and labelling, such that all ingredients, associated health harms, and warnings are listed (7). The Cancer Council and the National Heart Foundation recommend a ban on retail sale of non-nicotine e-cigarettes (21). Industry and supply-chain stakeholder objections citing economic causes and lack of data are on similar lines as for other domains. Considerations of respect for autonomy, self-determination as well as consumer rights imply that the provision of risk information through labelling is a necessity. Effective labelling, together with mass-media and social marketing interventions warning about the dangers of e-cigarettes, infringes on personal liberty the least, and needs to be considered as a public health intervention. This would fill an important policy gap and can be implemented in Australia, which has demonstrated its success in the space through successful implementation of plain packaging standards. It is also important to stress that warning alone, as many proponents of libertarianism might argue, is unlikely to have a major influence on usage of e-cigarettes. However, provision of information to citizens, through labelling is an ethical imperative for the government. This might be challenging considering the wide variety of e-cigarette products, and the consequent lack of certainty on their potential for harm in short and long-term users.

\section{Enforcing bans on e-advertising, promotion, and sponsorship of e-cigarettes}

Advertisements and marketing for tobacco products, including restrictions on internet advertising is enforced through the Tobacco Advertising Prohibition Act 1992 (29).

E-cigarettes without nicotine do not come under the purview of this Act and as such their marketing and advertising are largely unregulated, with industry employing the tactics of former tobacco marketing campaigns. The marketing objective is to associate e-cigarettes with glamour and desirable lifestyles. Analysis of e-cigarette promotion on social media highlights that it aims to show them as healthconducive devices, and many circumvent Australian tobacco control laws (30-32). Regulating social media however is challenging considering the borderless nature of the internet.

However, a bigger issue in the space is the targeting of youth. E-cigarettes are available in several flavours and are being specifically targeted at adolescents and young adults who find these flavours attractive (33). There is an increased concern around their ingestion by children, with even deaths being reported in the USA (19). Children lack autonomy in decision making and there is a need for instituting public health action to protect them. Apart from vulnerability, there are also concerns around long term harms of e-cigarette use in children and younger people. Proponents of e-cigarettes have for long claimed that the "gateway effect" has no credibility, but a recent meta-analysis of longitudinal studies with data from 17,389 adolescents and young adults, found that those who used e-cigarettes were more likely to initiate and use cigarettes (past 30-day use) than those who do not $(34,35)$. In Australia, current e-cigarette use has increased between 2016 and 2019 (3). Most of the increase is in younger people - from $6.8 \%$ to $18.7 \%$ in $18-24$ year olds, and from $3.6 \%$ to $13.7 \%$ in $25-29$-year olds (3). As such, a complete ban on e-cigarettes for protecting the public good of low tobacco consumption in Australia might be considered necessary (12).

\section{Raising taxes on e-cigarettes}

The e-cigarette industry has, over the last few years, been consolidated through mergers and acquisitions by tobacco companies(36). As such, "Big Tobacco" benefits from dual use as well as promoting themselves as agents of harm reduction. Such moral positioning has become imperative for the tobacco industry owing to the changed cultural discourse around tobacco use wherein it is no longer glamorised. As such, the industry discourse has been to show an interest in collaborating with government to help achieve "public health gains" while it can continue to contribute to the economy through tobacco taxes. Critics argue that it is immoral to contribute to the profits of tobacco industries which will continue to use the monies for promoting their products and harm more people (19). A "levelling-up" approach has been suggested wherein e-cigarette sales are allowed, with similar restrictions to those on cigarettes, and taxed at the same or a lower rate (since they are less harmful) (9). Proponents of e-cigarettes consider taxation as it enables distributive justice. 


\section{Discussion}

A comprehensive analysis across the spectrum, instead of a piecemeal ethical analysis, allowed for an understanding of the ethical tensions around e-cigarette use. The objections raised by peak medical bodies to e-cigarettes for smoking cessation will be challenged owing to the changing nature of evidence around it. Further randomised controlled trials in the Australian context might be mandated not only for the cause of science, but also to resolve the ethical conundrum around their long-term safety.

Apart from ethics, economic considerations influence decisions regarding the revision of taxation structures, and legislation on packing and labelling of e-cigarettes. While the current analysis does not aim to be prescriptive, further dialogue on non-nicotine containing e-cigarettes is necessary. With their role in smoking cessation being limited, they do serve the role of normalising and glamourising a "smoking culture" in the youth. At the core, however, lies the need for Australia to sustain and further its historic low in smoking prevalence. Australia, unlike many other countries, has been successful in bringing down tobacco consumption. With its geographic location, relatively homogenous culture and smaller population, policy implementation has been less challenging than in larger countries on several counts.

For many countries, economic considerations, illicit tobacco trade, and industry interference are major challenges (37-41) for tobacco control. For many low-and middle-income nations, the variety of tobacco products including smokeless varieties(42), further complicates the issue. In Australia, the low level of tobacco consumption is a public good that needs to be preserved through robust public policy.

\section{Conclusion}

Policies around e-cigarette control are topical globally, but these rarely involve a comprehensive analysis of ethical issues from a public health standpoint and using a valueneutral framework. As for example, the complete ban on ecigarettes in India (43) with almost no discussion on public health ethics (44). It is also useful to note that much of the evidence around e-cigarettes for smoking cessation tool might not be generalised to LMICs.

Ethics, evidence and context go hand-in-hand to inform public policy debates. The comprehensive analysis using a public health ethics framework, structured around an acceptable disease control framework, contextualised to a country, in this paper could be a useful exemplar for more analyses in other countries and on other public health issues.

'Note: A peak body in Australian parlance implies an association of industries or trade unions with allied interests.

\section{Disclaimer}

The opinions expressed in this article are those of the author and might not represent those of the institution.
Conflict of interest and funding: None declared.

\section{References}

1. Chapman S, Byrne F, Carter SM. "Australia is one of the darkest markets in the world": the global importance of Australian tobacco control. Tob Control. 2003;12 Suppl 3:iii 1-3.

2. Scollo M, Bayly M, Wakefield M. Plain packaging: a logical progression for tobacco control in one of the world's 'darkest markets'. Tob Control. 2015;24(Suppl 2):ii3-ii8.

3. Australian Institute of Health and Welfare. National Drug Strategy Household Survey 2019. Canberra:AlHW; 2020.

4. Watts C, Burton S, Freeman B. Creating a market for IQOS: analysis of Philip Morris' strategy to introduce heated tobacco products to the Australian consumer market. Tob Control. 2020.

5. Wodak A, Mendelsohn CP. The Australian approach to tobacco harm reduction is even more misguided than the US approach. Am J Public Health. 2020;110(6):783-4.

6. Hall W, Bonevski B, Gartner C. Policy-based evidence on e-cigarette, or vaping product, use-associated lung injury. Drug Alcohol Rev. 2020;39(4):426-7.

7. Erku DA, Morphett K, Steadman KJ, Gartner CE. Policy Debates Regarding Nicotine Vaping Products in Australia: A Qualitative Analysis of Submissions to a Government Inquiry from Health and Medical Organisations. Int J Environ Res Public Health. 2019;16(22).

8. McAlinden KD, Eapen MS, Lu W, Sharma P, Sohal SS. The rise of electronic nicotine delivery systems and the emergence of electronic-cigarette-driven disease. Am J Physiol Lung Cell Mol Physiol. 2020;319(4):L585-195.

9. Hall W, Gartner C, Forlini C. Ethical issues raised by a ban on the sale of electronic nicotine devices. Addiction. 2015;110(7):1061-7.

10. Thomas R, Parker LS, Shiffman S. The ethics of tobacco harm reduction: An analysis of e-cigarette availability from the perspectives of utilitarianism, bioethics, and public health ethics. Nicotine Tob Res. 2020.

11. Moore $M$, McKee $M$, Daube M. Harm reduction and e-cigarettes: Distorting the approach. J Public Health Policy. 2016;37(4):403-10.

12. Dawson A, Verweij M. No Smoke Without Fire: Harm Reduction, ECigarettes and the Smoking Endgame. Public Health Ethics. 2017;10(1): 1-4.

13. Ortmann LW, Barrett DH, Saenz C, Bernheim RG, Dawson A, Valentine JA, et al. Public Health Ethics: Global Cases, Practice,and Context. 2016. In: Public Health Ethics: Cases Spanning the Globe [Internet]. Cham $(\mathrm{CH})$ : Springer Open Public Health Ethics Analysis; 2016 Apr [cited 2020 Nov13] . Chapter '1. Available from: https:// www.ncbi.nlm.nih.gov/books/NBK435780/

14. Levy DT, Fong GT, Cummings KM, Borland $R$, Abrams DB, Villanti AC, et al. The need for a comprehensive framework. Addiction. 2017;112(1): 22-4.

15. World Health Organization. Report on the global tobacco epidemic, 2008: the MPOWER package. Geneva:WHO; 2008.

16. World Health Organization. WHO framework convention on tobacco control. Geneva:WHO; 2003.

17. Australian Bureau of Statistics. National Aboriginal and Torres Strait Islander Health Survey. Canberra: ABS 2019 [cited 2020 Nov 13]. Available from: https://www.abs.gov.au/statistics/people/aboriginaland-torres-strait-islander-peoples/national-aboriginal-and-torresstrait-islander-health-survey/2018-19.

18. Thurber KA, Walker J, Maddox R, Marmor A, Heris C, Banks E, et al. A review of evidence on the prevalence of and trends in cigarette and e-cigarette use by Aboriginal and Torres Strait Islander youth and adults. Canberra: The Australian National University; 2020.

19. Franck C, Filion KB, Kimmelman J, Grad R, Eisenberg MJ. Ethical considerations of e-cigarette use for tobacco harm reduction. Respir Res. 2016;17(1):53.

20. Epstein RA. The Harm Principle - And How It Grew. The University of Toronto Law Journal. 1995;45(4):369-417.

21. Cancer Council. E-cigarettes : what are they and are they harmful? Sydney: Cancer Council; 2020 [Available from: https:// www.cancer.org.au/cancer-information/causes-and-prevention/ smoking-and-tobacco/e-cigarettes

22. The Royal Australian College of General Practitioners; Supporting smoking cessation: A guide for health professionals. 2nd ed. Melbourne:RACGP; 2019.

23. Hartmann-Boyce J, McRobbie $H$, Lindson N, Bullen C, Begh R, Theodoulou A, et al. Electronic cigarettes for smoking cessation. Cochrane Database Syst Rev. 2020;10:Cd010216.

24. Hall W, Gartner C, Forlini C. Nuances in the ethical regulation of electronic nicotine delivery systems. Addiction. 2015;110(7):1074-5. 
25. Therapeutic Goods Administration. Interim decision of entry of nicotine in the Poisons Standard: Information for consumers. In: Administration TG, editor. Canberra: Ministry of Health; 2020.

26. Therapeutic Goods Administration. Prohibition on importing ecigarettes containing vaporiser nicotine. Canberra: Ministry of Health; 2020.

27. Clun R. Waiting to exhale: Ampol backs easing of e-cigarette restrictions. The Sydney Morning Herald. 2020 Nov 12

28. Jackson D. Industry calls for a delay to proposed restrictions on vaping product sales. Convenience \& Impulse Retailing. 2020 (col. 2020).

29. Tobacco Advertising Prohibition Act 1992 : Compilation No 13, No. 218, $1992(2016)$

30. McCausland K, Maycock B, Leaver T, Jancey J. The Messages Presented in Electronic Cigarette-Related Social Media Promotions and Discussion: Scoping Review. J Med Internet Res. 2019;21(2):e11953.

31. McCausland K, Maycock B, Leaver T, Wolf K, Freeman B, Thomson K, et al. E-Cigarette Promotion on Twitter in Australia: Content Analysis of Tweets. JMIR Public Health Surveill. 2020;6(4):e15577.

32. Burton S, Dadich A, Soboleva A. Promotion of JUUL on Twitter. J Adolesc Health. 2018;63(5):525-6.

33. Raj AT, Patil S, Gupta AA, Suveetha G. Flavored tobacco to Ecigarette's: How the tobacco industry sustains its product flow. Oral Oncol. 2018;85:110.

34. Soneji S, Barrington-Trimis JL, Wills TA, Leventhal AM, Unger JB, Gibson LA, et al. Association Between Initial Use of e-Cigarettes and Subsequent Cigarette Smoking Among Adolescents and Young Adults: A Systematic Review and Meta-analysis. JAMA Pediatr. 2017;171(8):788-97.

35. Soneji S. Errors in Data Input in Meta-analysis on Association Between
Initial Use of e-Cigarettes and Subsequent Cigarette Smoking Among Adolescents and Young Adults. JAMA Pediatr. 2018;172(1):92-3.

36. Houston AR, Howard A, Sweanor D. Placing the Legal Vape Market in the Hands of Big Tobacco. Am J Public Health. 2020;110(6):781-2.

37. Arora M, Datta P, Barman A, Sinha P, Munish VG, Bahl D, et al. The Indian Bidi Industry: Trends in Employment and Wage Differentials. Front Public Health. 2020;8:572638.

38. Nguyen HTT, Giang LT, Pham TN. Empirical analysis on the illicit trade of cigarettes in Vietnam. Tob Control. 2020;29(Suppl 4):s281-s6.

39. Evans-Reeves K, Hatchard J, Rowell A, Gilmore AB. Illicit tobacco trade is 'booming': UK newspaper coverage of data funded by transnational tobacco companies. Tob Control. 2020;29(e1):e78-e86.

40. van der Zee $\mathrm{K}$, Vellios $\mathrm{N}$, van Walbeek $\mathrm{C}$, Ross $\mathrm{H}$. The illicit cigarette market in six South African townships. Tob Control. 2020;29(Suppl 4):s267-s74.

41. Chugh A, Bassi S, Nazar GP, Bhojani U, Alexander C, Lal P, et al. Tobacco Industry Interference Index: Implementation of the World Health Organization's Framework Convention on Tobacco Control Article 5.3 in India. Asia Pac J Public Health. 2020;32(4):172-8.

42. Singh PK, Yadav A, Lal P, Sinha DN, Gupta PC, Swasticharan L, et al. Dual Burden of Smoked and Smokeless Tobacco Use in India, 20092017: A Repeated Cross-Sectional Analysis Based on Global Adult Tobacco Survey. Nicotine Tob Res. 2020;22(12):2196-202.

43. Vishal Rao US, Arakeri G, Ravishankar S, Kar A, Thakur S, Li RJ, et al. The E-cigarette ban in India-A step in the right direction? J Oral Pathol Med. 2020;49(7):617-20.

44. Indian Council of Medical Research. White Paper on Electronic Nicotine Delivery System. Indian J Med Res. 2019;149(5):574-83. 